\title{
Outcomes of adjuvant whole-brain radiotherapy versus hypofractionated stereotactic radiotherapy after surgical resection of brain metastases: a propensity score-matched analysis
}

\author{
Audrey Keller ${ }^{1}$, François Lefebvre ${ }^{2}$, Damien Ricard ${ }^{3}$, Georges Noël ${ }^{1,4}$, Delphine Antoni ${ }^{1,4}$ \\ ${ }^{1}$ Radiotherapy University Department, ICANS, 17, rue Albert Calmette 67200 Strasbourg, France; ${ }^{2}$ Laboratory of Biostatistics, Faculté de Médecine, \\ 4, rue Kirschleger, 67085 Strasbourg Cedex, France; ${ }^{3}$ Department of Neurology, Hôpital d'Instruction des Armées Percy, Service de Santé des \\ Armées, 1 Rue du Lieutenant Raoul Batany, 92190 Clamart, France; ${ }^{4}$ Radiobiology Laboratory, EA 3430, Federation of Translational Medicine of \\ Strasbourg (FMTS), University of Strasbourg, Strasbourg, France \\ Contributions: (I) Conception and design: A Keller, G Noël, D Antoni; (II) Administrative support: G Noël; (III) Provision of study materials or \\ patients: A Keller, G Noël, D Antoni; (IV) Collection and assembly of data: A Keller, D Antoni; (V) Data analysis and interpretation: A Keller, F \\ Lefebvre, G Noël, Delphine Antoni; (VI) Manuscript writing: All authors; (VII) Final approval of manuscript: All authors. \\ Correspondence to: Prof. Georges Noël, MD, PhD. Radiotherapy University Department, ICANS, 17, rue Albert Calmette, 67200 Strasbourg, France. \\ Email: g.noel@icans.eu.
}

Background: To assess the risks of local and distant failure and overall survival time in patients treated with hypofractionated stereotactic radiotherapy (HFSRT) to the postoperative cavity for brain metastases (BMs) compared with patients treated with adjuvant whole-brain radiation therapy (WBRT).

Methods: Between July 2005 and February 2015, 196 non-randomized patients with 202 resected BMs were treated with post-operative WBRT or HFSRT at a single institution. The propensity score was included as a covariate to compare the interval to local failure, distant brain failure and overall survival time. The matching covariates consisted of the age, Karnofsky performance status, primary disease, number of BMs, extracranial disease status and presence or absence of extracranial metastases.

Results: In total, 110 patients (54.5\%) received adjuvant WBRT, and 92 HFSRT procedures (45.5\%) were delivered after surgery. A Cox model adjusted on the propensity score showed that the brain distant failure was significantly associated with treatment modality. Compared with WBRT, the patients who received HFSRT had a significantly greater risk of developing distant brain recurrence [HR =3.37 (2.13-5.33), $\mathrm{P}<0.001]$. No difference was observed in local failure $(\mathrm{HR}=1.16, \mathrm{P}=0.77)$ between the groups. In the propensity-matched cohort, the effect of treatment on survival was not significant $(\mathrm{P}=0.14)$, but it depended on the time. Within the first 20 months, the patients treated with WBRT had a 2 times higher risk of death than did patients treated with HFSRT [HR =2.17 (1.42-3.32), P=0.0003].

Conclusions: Compared with the standard WBRT after the resection of BMs, stereotactic radiotherapy to the surgical bed produced a comparable local control rate to that of WBRT with a survival advantage in the first 20 months.

Keywords: Whole brain radiation therapy; stereotactic radiotherapy; surgical bed; brain metastases (BMs); propensity score

Submitted Dec 17, 2019. Accepted for publication Jun 01, 2020.

doi: $10.21037 /$ cco-19-269

View this article at: http://dx.doi.org/10.21037/cco-19-269 


\section{Introduction}

Brain metastases (BMs) occur in $20 \%$ to $30 \%$ of all cancer patients (1). Patient's outcome with BMs is generally poor, with median survivals after whole-brain radiation therapy (WBRT) alone in the range of 3 to 6 months (2). Removal of brain metastasis remains the gold standard in several situations. However, surgery only of BMs resulted in unacceptably high relapse rates in the surgical bed and/ or at new sites in the brain (42\%) (3). For two decades, the standard of care for years following the surgical resection of BMs has been post-operative WBRT; Patchell et al. described a local and distant disease control advantage for WBRT after the resection of a solitary metastasis, but the trial was not statistically powered and did not show an overall survival (OS) benefit (4). This has gradually led to a new treatment paradigm, namely to perform stereotactic radiotherapy (SRT) of the tumor bed to improve local control with deferring WBRT. A review of 21 retrospective series, including 1,011 patients, showed promising results after such management; the crude one-year control rate in the tumor bed was $79 \%$ with $51 \%$ of patients developing new BMs $(5,6)$. Additionally, delayed white matter toxicity, detected by magnetic resonance imaging (MRI), occurs more often after WBRT than after SRT, leading to postpone WBRT late (7). A large recent retrospective study of two French centers showed the efficacy and the good tolerance of postoperative fractionated SRT in tumor bed (8). Results of the multi-institutional randomized trial (NCCTG-N107C) was recently published and demonstrated the efficacy of stereotactic radiosurgery (SRS) compared with WBRT in terms of prevention of cognitive side-effects. There was no significant difference in survival, and local control of operated metastases and brain control were worst after stereotactic treatment than after WBRT (9). Furthermore, a second randomized trial showed that stereotactic irradiation of the operative bed was not inferior to WBRT in terms of local relapse but neurological death and overall survival were worst in the stereotactic group compared to the WBRT group (10). Consequently, the impact of postoperative stereotactic irradiation on overall survival remains questioned.

At our single institution, we have previously studied the role of surgery and postoperative WBRT, in which surgical resection followed by WBRT produced a better outcome than did WBRT alone for RPA I or II patients with 1 or 2 BMs (11). In the present report, we compared the clinical outcomes of adjuvant hypofractionated stereotactic radiotherapy (HFSRT) whose results were previously published (8) with those of WBRT after the resection of BMs. To perform a comparative analysis of this nonrandomized patient cohort, we used propensity score-based analysis.

We present the following article in accordance with the STROBE reporting checklist. Available at http://dx.doi. org/10.21037/cco-19-269.

\section{Methods}

We retrospectively reviewed 202 patient data and radiotherapy procedures (WBRT, $\mathrm{n}=110$; HFSRT, $\mathrm{n}=92$ ) following the neurosurgical resection of BMs delivered between July 2005 and February 2015. One hundred ninetysix patients with 1-3 metastases and Recursive Partitioning Analysis (RPA) Class I to III were included in our study. In the group surgery followed by HFSRT, patients who had undergone a previous WBRT were excluded from the analysis. Local failure (LF) was defined as new or increased contrast enhancement involving the resection cavity on MRI. Distant brain failure (DBF) was defined as the presence of new enhancing BMs or leptomeningeal disease outside the postoperative cavity.

\section{Radiotherapy}

Between July 2005 and January 2011, 110 patients underwent WBRT with different schedules according to the RPA Class, prescribed with a boost to the resection cavity. For RPA I patients, a dose of 40 Gy in 20 fractions of 2 Gy (5 fractions per week) was delivered in the whole brain, with a radiation boost on the operative site at a dose of $16 \mathrm{~Gy}$ in 8 fractions of $2 \mathrm{~Gy}$. Another schedule was also used with a dose of 37.5 Gy in 15 fractions of 2.5 Gy, followed by a boost at the metastatic site (10 Gy in 4 fractions of 2.5 Gy). For RPA class II and III patients, a dose of 30 Gy in 10 fractions of 3 Gy was delivered, with a boost of $9 \mathrm{~Gy}$ in 3 fractions of 3 Gy. WBRT was performed with 6-MV photons of a linear accelerator, using bilateral parallel fields. The radiation boost on the operative site was delivered with 6 or $25 \mathrm{MV}$ after 3-D treatment planning.

Between February 2012 and February 2015, 86 patients with 92 cavities were treated with stereotactic radiotherapy to the resection cavity of BMs. HFSRT was delivered in 3 fractions of 7.7 Gy every 2 days (i.e., d1-3-5) prescribed to the $70 \%$ isodose line. A Novalis $\mathrm{Tx}^{\mathrm{TM}}$ (Varian Medical 
Systems ${ }^{\circledR}$, Palo Alto, CA, USA) linear accelerator was used in all cases. The clinical target volume (CTV) was designated as the entirety of the resection cavity, including any residual enhancing disease, and the planning target volume (PTV) was defined by an automatic extension of CTV with a 2-mm isotropic margin. Treatments were delivered using dynamic conformal arcs or Volumetric Modulated Arc Therapy (VMAT). HFSRT was planned using the Treatment Planning System (TPS) iPlan ${ }^{\circledR}$ RT Dose (Brainlab ${ }^{\circledR}$ AG, Feldkirchen, Germany) for arc therapy or the Eclipse ${ }^{\circledR}$ System (Varian Medical Systems ${ }^{\circledR}$, Palo Alto, CA, USA) for VMAT planning.

\section{Statistical analysis}

The baseline characteristics of patients were assessed using descriptive statistics, including the median and range for continuous variables or frequencies and percentages for categorical variables. The end points of this study were local failure-free survival, distant brain failure-free survival and OS. All intervals were calculated from the date of the end of HFSRT or WBRT. Survival curves were carried out using the Kaplan-Meier method and Cox model.

A Propensity Score (PS), defined as the conditional probability of receiving treatment given individual's covariates, was used to balance the covariates in the two groups, thereby reducing the biases $(12,13)$. It was used to consider the baseline characteristics of the patients. Thus, the covariates consisted of the age, Karnofsky performance status (KPS), primary disease, number of BMs, status of extracranial disease and presence or absence of extracranial metastases. Cox models for competing risks and survival were performed to compare the two treatments, considering the probability of receiving each treatment with the propensity score. The robustness of fit was checked with the test of Harrell (14), and time-dependent covariates were added when necessary. Sub-group models were also performed to determine the effect of treatment over time. A two-sided $\mathrm{P}$ value less than 0.05 was taken as statistically significant. All computations were conducted in R, version 3.2.2.

This monocentric retrospective study complies with the "reference methodology" MR004 adopted by the French Data Protection Authority (CNIL) and patients did not object to the use of their clinical data for the research purpose. Written informed consent was obtained from the patient for publication of this study and any accompanying images. A copy of the written consent is available for review by the Editor-in-Chief of this journal.

\section{Results}

\section{Baseline characteristics in the entire patient cobort}

The median follow-up time of the entire cohort was 14 months (0-71.6). The median age of the patients was 59.3 years (26.2-82.3), and the male to female ratio was 1.2 , with 111 males and 91 females. The RPA class distribution was as follows: RPA I ( $\mathrm{n}=34,16.8 \%)$, RPA II ( $\mathrm{n}=158,78.2 \%)$ and RPA III $(\mathrm{n}=10,4.9 \%)$. The patient characteristics are summarized in Table 1 . In total, 110 patients (54.5\%) received WBRT, and 92 HFSRT procedures (45.5\%) in 86 patients were delivered following surgical resection. The mean time from surgery to radiotherapy was 50 days (16-92.9, median 45 days). GPA, number of metastases and extracranial metastases were significantly unfavorable in the HFSRT group compared to the WBRT group $(\mathrm{P}=0.02$, $\mathrm{P}=0.037$ and $\mathrm{P}=0.03$, respectively).

The median OS was 15.3 months (95\% CI, 11.7-19.0). At the end of the follow-up period, 59 patients were still alive. The 6-, 12-, and 24-month OS rates for the entire group were $74.1 \%(71-77.2 \%), 56.7 \%(53.2-60.2 \%)$ and $35.1 \%$ (31.6-38.6\%), respectively. Of the 137 patients who died, $44(32.1 \%)$ succumbed to their extra-cranial disease, $40(29.2 \%)$ died of progressive intracranial disease, 24 patients $(17.5 \%)$ died of other causes and 29 causes of death (21.2\%) were unknown (Table 2).

\section{Results in the entire patient cohort and in the propensity score-matched cohort}

The median follow-up time was 14.5 months $(0-71.6)$ and 12.9 months $(0.89-40.5)$ in the WBRT and HFSRT groups, respectively.

After the combined treatment of surgery plus radiotherapy, 77 (39.3\%) developed distant brain failure (DBF). The crude DBF rates were $25.4 \%$ of cases in the WBRT group and $55.4 \%$ of the cases in the HFSRT group $[\mathrm{HR}=3.37$ (2.13-5.33), $\mathrm{P}<0.001]$ (Figure 1).

After the combined treatment of surgery plus radiotherapy, 21 patients (10.4\%) developed local failure (LF) at the surgical site. The crude LF rates were $10 \%$ after surgery + WBRT, and $13 \%$ after surgery + HFSRT [HR $=1.16(0.43-3.09), \mathrm{P}=0.77$ ] (Figure 2).

The 6-, 12-, and 24-month OS rates for the WBRT group were $73.1 \%(68.8-77.4 \%), 58.3 \%(53.6-63 \%)$ and 
Table 1 Patients characteristics

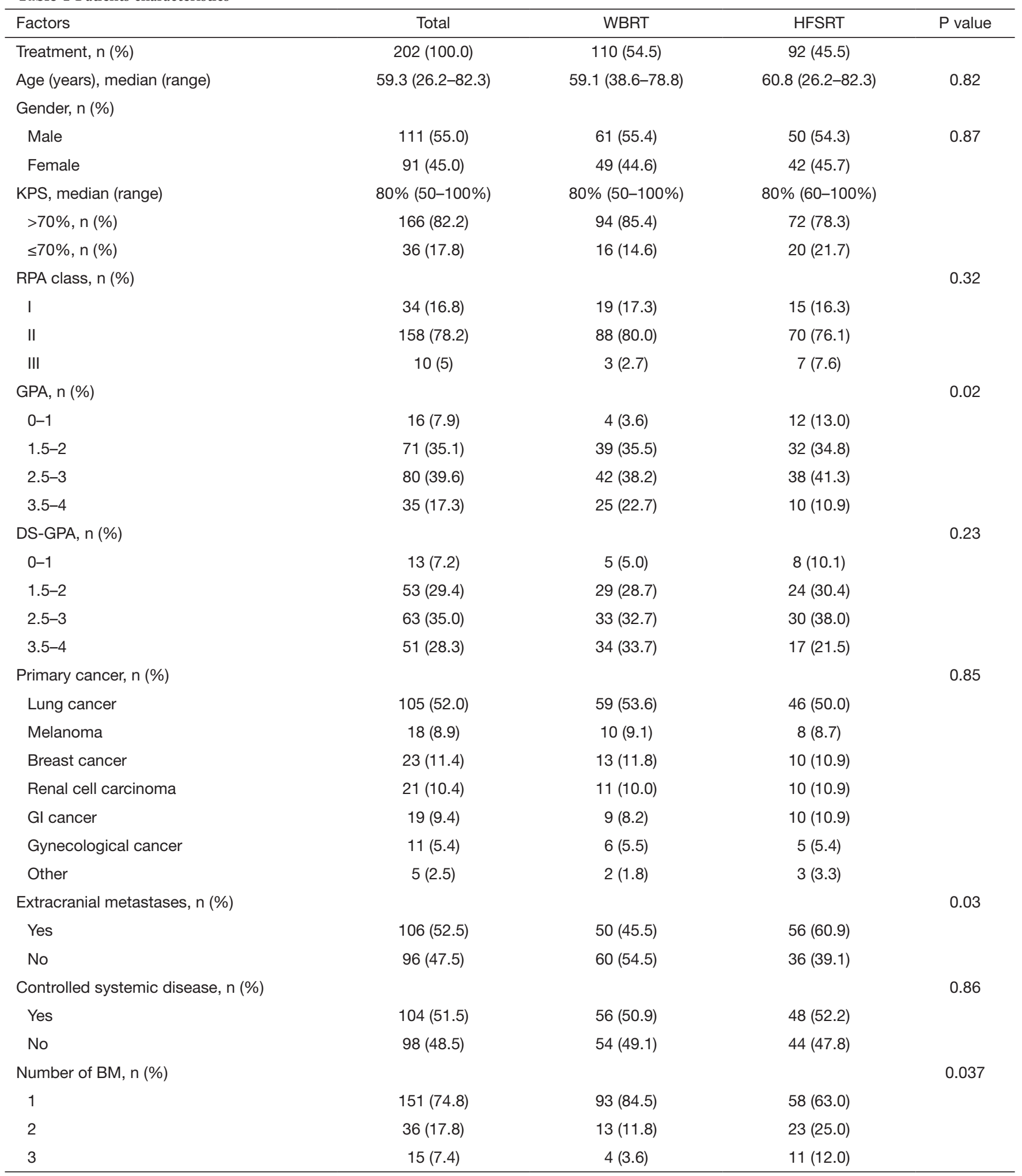

KPS, Karnofsky performance status; GPA, Graded Prognostic Assessment score; DS-GPA, Diagnosis-Specific GPA. 
Table 2 Causes of death

\begin{tabular}{lccc}
\hline Causes of death & Total & WBRT & HFSRT \\
\hline Total deaths & $137 / 196(70 \%)$ & $74 / 110(67 \%)$ & $63 / 86(73 \%)$ \\
Neurological cause & $40(29.2 \%)$ & $17(23 \%)$ & $23(36.5 \%)$ \\
Systemic disease & $44(32.1 \%)$ & $16(21.6 \%)$ & $28(44.4 \%)$ \\
Other & $24(17.5 \%)$ & $15(20.3 \%)$ & $9(14.3 \%)$ \\
Unknown & $29(21.2 \%)$ & $26(35.1 \%)$ & $3(4.8 \%)$ \\
\hline
\end{tabular}

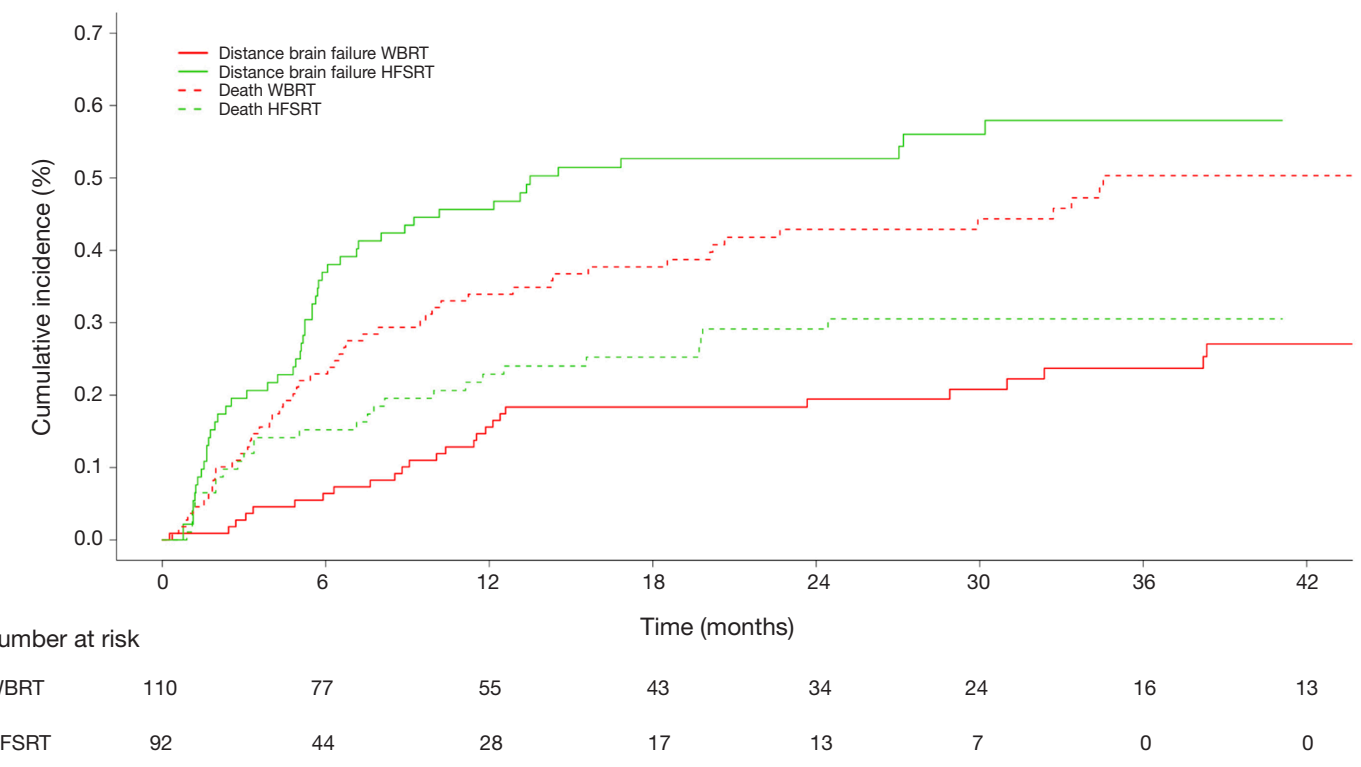

Figure 1 Time to distance brain failure since the treatment (months) $(\mathrm{P}<0.001)$.

$43.5 \%$ (38.6-48.4\%), respectively. For the HFSRT group, the 6-, 12-, 24-month OS rates were 76.1\% (71.7-80.5\%), $55.4 \%(50.2-60.6 \%)$ and $24.8 \%(20-29.6 \%)$, respectively. The Cox model adjusted for the propensity score showed that distant brain failure was significantly associated with treatment modality. In the propensity-matched cohort, the effect of treatment on survival was not significant $(\mathrm{P}=0.14)$ (Figure 3A), but it depended on the time. Within the first 20 months, patients treated with adjuvant WBRT had a 2.17 times higher risk of death than patients treated with HFRST [HR =2.17 (1.42-3.32), $\mathrm{P}=0.0003$ ] (Figure 3B). The causes of death in both groups are summarized in Table 2 .

\section{Discussion}

Recent data have shown that in patients with a solitary brain metastasis or oligometastatic disease, WBRT plus
SRS offers improved intracranial control, but not a survival benefit, compared with SRS alone $(3,15,16)$. Thus, patients with limited BMs can be treated with SRT, with the option of deferring the use of WBRT $(17,18)$. This strategy has been extrapolated to the treatment of patients with resected lesions $(5,8,19)$. A recent phase III trial demonstrated the efficacy of SRS with the same OS and less impact on cognitive function and the quality of life compared with WBRT in a post-operative setting (9).

\section{Local control}

In our study, HFSRT to the resection cavity produced rates of local tumor control that were comparable to those of WBRT after the resection of a metastatic tumor, and the Cox model adjusted for the propensity score exhibited no difference in local failure [ $\mathrm{HR}=1.16(0.43-3.09), \mathrm{P}=0.77$ ] 


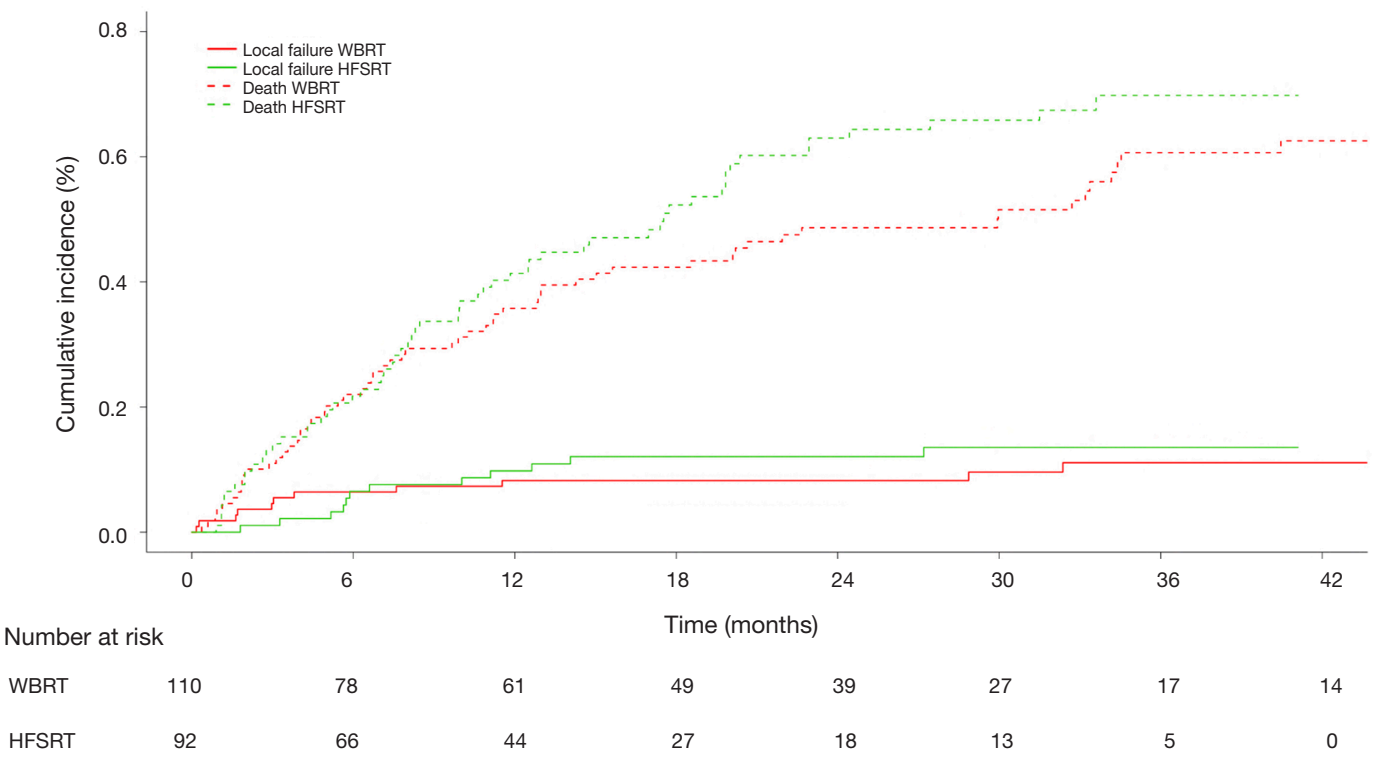

Figure 2 Time to local failure since the treatment (months) $(\mathrm{P}=0.77)$.

between the groups. This result supports the conclusion that stereotactic radiotherapy to the resection cavity is an effective treatment for the management of resected BMs. Studies have reported 1-year LC rates of $73 \%$ to $100 \%$ after single-dose adjuvant SRS (20-22). Similar numbers have been reported for multi-fractionated SRS using up to 5 fractions (8,23-25). In the meta-analysis of Gans et al., which included 14 studies of post-operative SRS, the mean crude local control was $83 \%$ (5). Similarly, in the recent trial of Mahajan et al., the patients were randomized to either SRS or observation of the surgical cavity. The LC rates were superior in the SRS group versus the observation group (HR $=0.46,95 \% \mathrm{CI}: 0.25-0.85, \mathrm{P}=0.011$ ), with LC rates of $72 \%$ vs. $45 \%$ at 12 months, respectively (26). In comparison, in the randomized trial of Kocher et al., adjuvant WBRT after surgery was shown to have a 2-year local control rate of $73 \%$ (3).

Although adjuvant WBRT may be effective for improving local control (LC), significant neurocognitive deficit and memory dysfunction have been reported $(16,27)$.

\section{Controversy}

Controversy surrounds the issue of cognitive and neurologic preservation, specifically regarding the relative benefit and risk of WBRT versus those of focused radiation for patients with a limited number of metastases. Indeed, the potential complication of the cognitive decline after
WBRT must be weighed against the risk of developing additional intracranial metastasis. In fact, one of the benefits of WBRT is the reduction of the appearance of new intracranial metastases. In our series, patients who received HFSRT after surgery had a significantly greater risk of developing distant brain recurrence $[\mathrm{HR}=3.37$ (2.13-5.33), $\mathrm{P}<0.001]$ than did patients with adjuvant WBRT. Regine et al. described an increasingly significant risk of brain tumor failure after the use of SRS alone, $70 \%$ of patients developed symptoms at the time of recurrence, and 59\% of them had an associated neurologic deficit (28). In a study by Aoyama et al., 132 patients with 1-4 BMs were randomized between WBRT + SRS and SRS alone. The average duration until deterioration was 16.5 months in the WBRT+SRS group versus 7.6 months in the SRS alone group, with a statistical difference $(\mathrm{P}=0.05)$. They concluded that control of the brain tumor was the most important factor for stabilizing neurocognitive function (29). However, stereotactic radiotherapy is repeatable if relapse occurs in another brain area (30), and salvage WBRT remains always possible. Thus, close MRI follow-up is mandatory for detecting and treating small metastases, when the risk of neurocognitive deterioration is low.

Moreover, although adjuvant WBRT may be effective to improve local and distant control, a significant neurocognitive deficit and memory dysfunction have been reported after this strategy approach. The randomized trial conducted by Chang et al. recruited 58 patients and was 


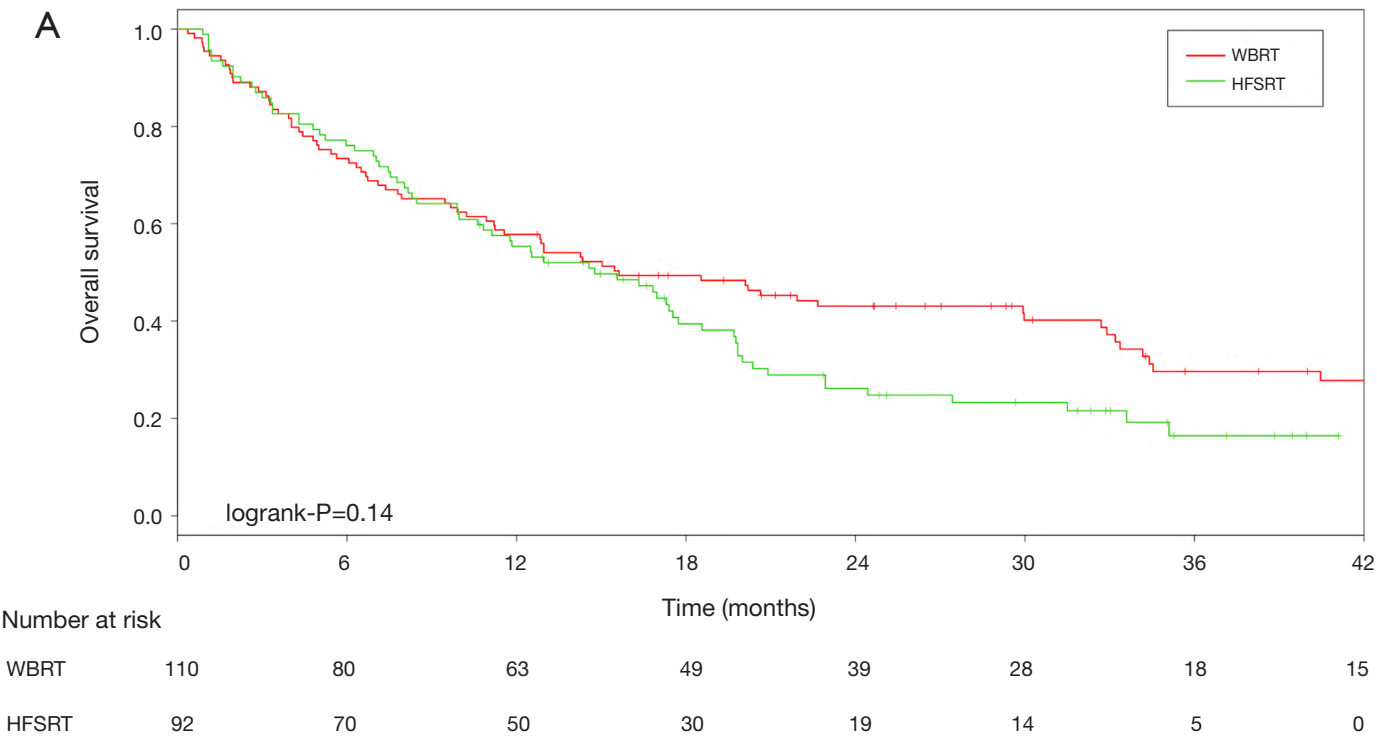

B

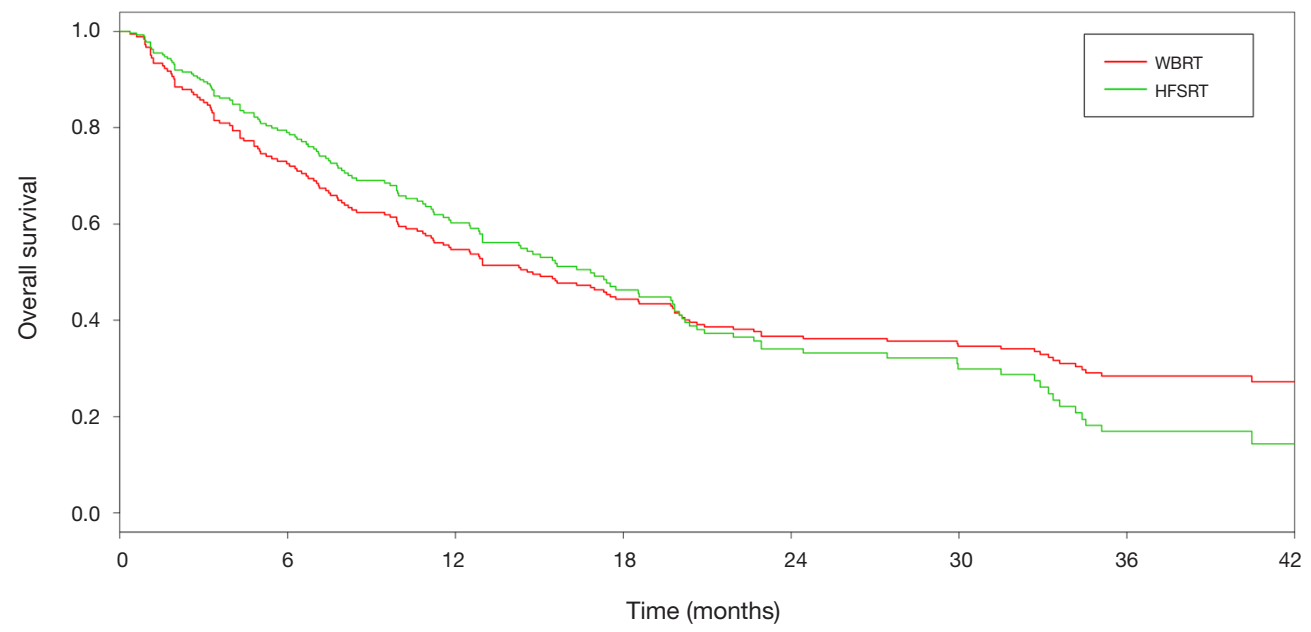

Figure 3 (A) Overall survival since the treatment (months) ( $\mathrm{P}=0.14$ ); (B) overall survival in the first 20 months, since the treatment (months) $(\mathrm{P}=0.0003)$.

stopped prematurely. In fact, an interim analysis revealed that the deterioration in learning and memory function at 4 months after treatment was significantly more frequent among the patients who received WBRT + SRS than among those who received SRS alone (16). In the study of Brown et al., 213 patients with 1-3 metastases were randomized between SRS alone and SRS + WBRT, and less cognitive deterioration was observed at 3 months in the SRS alone group than in the SRS + WBRT group $(\mathrm{P}<0.001)$. Furthermore, the quality of life was higher at 3 months with SRS alone $(\mathrm{P}=0.001)$. However, there was no statistically significant difference in the overall survival between the groups $(\mathrm{P}=0.92)$ (27).

Finally, the result of the recent multi-institutional trial NCCTG-N107C found comparable survival $(\mathrm{P}=0.65)$, less cognitive decline [cognitive deterioration at 6 months: 85.7\% after WBRT and $53.8 \%$ after SRS $(\mathrm{P}=0.0006)]$ and better quality of life following SRS versus whole brain radiotherapy after resection. Thus, post-operative SRS has become the new standard of care for patients with resected BMs (9).

One limitation of our study is that the retrospective 
nature of the data collection was not allowed to identify exhaustively the clinical and neurocognitive evolution of patients treated with radiation. In our propensitymatched cohort, the effect of treatment on survival was not significant $(\mathrm{P}=0.14)$, but it depended on the time. Within the first 20 months, the patients treated with WBRT after surgery had a 2 times higher risk of death than patients treated with adjuvant HFSRT to the surgical bed [HR $=2.17$ (1.42-3.32), $\mathrm{P}=0.0003]$. Furthermore, this survival benefit was observed, despite an increased risk of relapse in the whole brain after HFSRT alone. Both groups were comparable because patients were matched based on the age, Karnofsky performance status, primary disease, number of BMs, status of extracranial disease and presence or absence of extracranial metastases, the main prognostic factors of OS. Indeed, there are several prognostic indices for patients with BMs: the Radiation Therapy Oncology Group's (RTOG) Recursive Partitioning Analysis (RPA) by Gaspar et al. (31), Graded Prognostic Assessment (GPA) developed from a database of 1,960 patients (32), and a recent diagnosis-specific prognostic index, the DS-GPA, based on a multi-institutional retrospective analysis of 4,259 other patients with BMs from breast carcinoma, small-cell and non-small cell lung carcinoma, GI cancers, melanoma, and renal cell carcinoma (33). This recent index is the sum of the scores for each of, maximally, four prognostic factors (age, KPS, extracranial metastases and number of BMs), adapted to the histology. Thus, it was important to match our patients on these same prognostic factors.

In our analysis, there was a survival benefit within the first 20 months for patients treated with adjuvant HFSRT. Several explanations could be given for this difference in survival. First, the deaths could be attributed directly to the treatment, specifically WBRT. However, $23 \%$ of the patients died of a neurological cause in the WBRT group, as did $36.5 \%$ of those in the HFSRT group, but we cannot definitively conclude because many of the patients in the WBRT group died of an unknown cause $(35.1 \%$ vs. $4.8 \%$ in the HFSRT group).

Otherwise, WBRT induced a delay in the delivery of systemic therapy during the weeks of administration of radiotherapy. A longer time without systemic therapy can cause the progression of the primitive tumor or even a seeding of tumor cells, with a potential impact on survival. HFSRT may be particularly important to avoid these delays for patients with newly diagnosed primary tumors or progressing systemic disease.

Another explanation is that several weeks after the administration of WBRT, patients continued to recover from its acute side effects: fatigue, somnolence syndrome, and transient cognitive impairment $(34,35)$. It is possible that other complications occurred during this worsening of the general state. It should also be noted that the populations were comparable at the time of radiation therapy, in terms of Karnofsky Performance Status (median KPS $80 \%$ ) or DS-GPA score $(\mathrm{P}=0.23)$. Moreover, the Graded Prognostic Assessment (GPA) was slightly better in the WBRT group than in the HFSRT group $(\mathrm{P}=0.02)$. Thus, patients treated with WBRT did not have a worse prognosis than patients treated with HFSRT, which cannot explain the differential survival rate.

Finally, and maybe a bias in our study, the two groups of patients were treated in two different periods (between 2005 and 2011 for WBRT and from 2012 to 2015 for HFSRT). The systemic therapies, which were not considered in our analysis, were probably different. In fact, the targeted therapies that are now transforming the treatment of cancer have changed the chances for some cancer patients drastically. Progress has been achieved, particularly in the treatment of lung cancer, which is the most represented population in our study (52\%) (36).

However, in this context of increased control of systemic disease, patients had a higher risk to develop brain recurrences and had a potentially higher risk to die from their intracranial disease, particularly in the HFSRT group, in which the risk of developing intracranial metastases is more important. Thereby, caution is still advised.

\section{Propensity score}

Propensity score matching is a robust method that attempts to estimate the effect of a treatment by accounting for the covariates that predict receiving the treatment to reduce the bias due to confounding variables. For retrospective studies, the assignment of each treatment is not possible, and propensity score matching attempts to mimic randomization. Evidence-based medicine depends on well-designed and conducted research, but patients are often included according to very specific criteria, and these selected populations do not always reflect reality. Furthermore, the inclusion in a randomized trial can be long, and the technical progress and innovations seem to proceed ahead of the clinical trial initiatives. Indeed, evidence-based medicine can be surpassed by the evolution of technology, so the new treatment can take the place of the standard of care, without proof of a trial. To gain speed (evidence-based 
care is increasingly complex and rapidly changing) and power (a small population limits the ability to test research hypotheses with sufficient power to draw conclusions), the experience at a single center level could increase with the development of big data $(37,38)$.

\section{Conclusions}

Stereotactic radiotherapy to the resection cavity is an effective treatment in the management of resected BMs, with comparable local control rates of adjuvant whole-brain radiotherapy. Unsurprisingly, patients who received focused therapy in the surgical bed had a significantly greater risk of developing distant brain recurrence than patients treated with WBRT. However, this increased risk did not affect patient survival. By contrast, a survival advantage was observed in the HFSRT group in first 20 months. The use of the propensity score has brought a statistical power of this retrospective series.

\section{Acknowledgments}

Funding: None.

\section{Footnote}

Reporting Checklist: The authors have completed the STROBE reporting checklist. Available at http://dx.doi. org/10.21037/cco-19-269

Data Sharing Statement: Available at http://dx.doi. org/10.21037/cco-19-269

Conflicts of Interest: All authors have completed the ICMJE uniform disclosure form (available at http://dx.doi. org/10.21037/cco-19-269). The authors have no conflicts of interest to declare.

Ethical Statement: The authors are accountable for all aspects of the work in ensuring that questions related to the accuracy or integrity of any part of the work are appropriately investigated and resolved. The trial was conducted in accordance with the Declaration of Helsinki. This monocentric retrospective study complies with the "reference methodology" MR004 adopted by the French Data Protection Authority (CNIL) and patients did not object to the use of their clinical data for the research purpose. Written informed consent was obtained from the patient for publication of this study and any accompanying images. A copy of the written consent is available for review by the Editor-in-Chief of this journal.

Open Access Statement: This is an Open Access article distributed in accordance with the Creative Commons Attribution-NonCommercial-NoDerivs 4.0 International License (CC BY-NC-ND 4.0), which permits the noncommercial replication and distribution of the article with the strict proviso that no changes or edits are made and the original work is properly cited (including links to both the formal publication through the relevant DOI and the license). See: https://creativecommons.org/licenses/by-nc-nd/4.0/.

\section{References}

1. Patchell RA. The management of brain metastases. Cancer Treat Rev 2003;29:533-40.

2. Horton J, Baxter DH, Olson KB. The management of metastases to the brain by irradiation and corticosteroids. Am J Roentgenol Radium Ther Nucl Med 1971;111:334-6.

3. Kocher M, Soffietti R, Abacioglu U, et al. Adjuvant wholebrain radiotherapy versus observation after radiosurgery or surgical resection of one to three cerebral metastases: results of the EORTC 22952-26001 study. J Clin Oncol 2011;29:134-41.

4. Patchell RA, Tibbs PA, Regine WF, et al. Postoperative radiotherapy in the treatment of single metastases to the brain: a randomized trial. JAMA 1998;280:1485-9.

5. Gans JH, Raper DMS, Shah AH, et al. The Role of Radiosurgery to the Tumor Bed After Resection of Brain Metastases: Neurosurgery 2013;72:317-25.

6. Roberge D, Parney I, Brown PD. Radiosurgery to the postoperative surgical cavity: who needs evidence? Int $\mathrm{J}$ Radiat Oncol Biol Phys 2012;83:486-93.

7. Stokes TB, Niranjan A, Kano H, et al. White matter changes in breast cancer brain metastases patients who undergo radiosurgery alone compared to whole brain radiation therapy plus radiosurgery. J Neurooncol 2015;121:583-90.

8. Keller A, Doré M, Cebula H, et al. Hypofractionated Stereotactic Radiation Therapy to the Resection Bed for Intracranial Metastases. Int J Radiat Oncol Biol Phys 2017;99:1179-89.

9. Brown PD, Ballman KV, Cerhan JH, et al. Postoperative stereotactic radiosurgery compared with whole brain radiotherapy for resected metastatic brain disease (NCCTG N107C/CEC.3): a multicentre, randomised, 
controlled, phase 3 trial. Lancet Oncol 2017;18:1049-60.

10. Kępka L, Tyc-Szczepaniak D, Bujko K, et al. Stereotactic radiotherapy of the tumor bed compared to whole brain radiotherapy after surgery of single brain metastasis: Results from a randomized trial. Radiother Oncol 2016;121:217-24.

11. Antoni D, Kehrli P, Clavier JB, et al. Outcome Improvement in RPA I or II Patients With 1 or 2 Brain Metastases by Combined Surgery and Radiotherapy. World J Oncol 2013;4:37-45.

12. D'Agostino RB. Propensity score methods for bias reduction in the comparison of a treatment to a nonrandomized control group. Stat Med 1998;17:2265-81.

13. Rosenbaum PR, Rubin DB. The central role of the propensity score in observational studies for causal effects. Biometrika 1983;70:41-55.

14. Grambsch PM, Therneau TM. Proportional hazards tests and diagnostics based on weighted residuals. Biometrika 1994;81:515-26.

15. Aoyama H, Shirato H, Tago M, et al. Stereotactic radiosurgery plus whole-brain radiation therapy vs stereotactic radiosurgery alone for treatment of brain metastases: a randomized controlled trial. JAMA 2006;295:2483.

16. Chang EL, Wefel JS, Hess KR, et al. Neurocognition in patients with brain metastases treated with radiosurgery or radiosurgery plus whole-brain irradiation: a randomised controlled trial. Lancet Oncol 2009;10:1037-44.

17. Linskey ME, Andrews DW, Asher AL, et al. The role of stereotactic radiosurgery in the management of patients with newly diagnosed brain metastases: a systematic review and evidence-based clinical practice guideline. J Neurooncol 2010;96:45-68.

18. Tsao MN, Rades D, Wirth A, et al. Radiotherapeutic and surgical management for newly diagnosed brain metastasis(es): An American Society for Radiation Oncology evidence-based guideline. Pract Radiat Oncol 2012;2:210-25.

19. Brennan C, Yang TJ, Hilden P, et al. A phase 2 trial of stereotactic radiosurgery boost after surgical resection for brain metastases. Int J Radiat Oncol Biol Phys 2014;88:130-6.

20. Hwang SW, Abozed MM, Hale A, et al. Adjuvant Gamma Knife radiosurgery following surgical resection of brain metastases: a 9-year retrospective cohort study. J Neurooncol 2010;98:77-82.

21. Jensen CA, Chan MD, McCoy TP, et al. Cavity-directed radiosurgery as adjuvant therapy after resection of a brain metastasis. J Neurosurg 2011;114:1585-91.

22. Mathieu D, Kondziolka D, Flickinger JC, et al. Tumor bed radiosurgery after resection of cerebral metastases. Neurosurgery 2008;62:817-23; discussion 823-824.

23. Ahmed KA, Freilich JM, Abuodeh Y, et al. Fractionated stereotactic radiotherapy to the post-operative cavity for radioresistant and radiosensitive brain metastases. J Neurooncol 2014;118:179-86.

24. Minniti G, Esposito V, Clarke E, et al. Multidose stereotactic radiosurgery $(9 \mathrm{~Gy} \times 3)$ of the postoperative resection cavity for treatment of large brain metastases. Int J Radiat Oncol Biol Phys 2013;86:623-9.

25. Wang CC, Floyd SR, Chang CH, et al. Cyberknife hypofractionated stereotactic radiosurgery (HSRS) of resection cavity after excision of large cerebral metastasis: efficacy and safety of an $800 \mathrm{cGy} \times 3$ daily fractions regimen. J Neurooncol 2012;106:601-10.

26. Mahajan A, Ahmed S, McAleer MF, et al. Post-operative stereotactic radiosurgery versus observation for completely resected brain metastases: a single-centre, randomised, controlled, phase 3 trial. Lancet Oncol 2017;18:1040-8.

27. Brown PD, Jaeckle K, Ballman KV, et al. Effect of Radiosurgery Alone vs Radiosurgery With Whole Brain Radiation Therapy on Cognitive Function in Patients With 1 to 3 Brain Metastases: A Randomized Clinical Trial. JAMA 2016;316:401-9.

28. Regine WF, Huhn JL, Patchell RA, et al. Risk of symptomatic brain tumor recurrence and neurologic deficit after radiosurgery alone in patients with newly diagnosed brain metastases: results and implications. Int J Radiat Oncol Biol Phys 2002;52:333-8.

29. Aoyama H, Tago M, Kato N, et al. Neurocognitive function of patients with brain metastasis who received either whole brain radiotherapy plus stereotactic radiosurgery or radiosurgery alone. Int J Radiat Oncol Biol Phys 2007;68:1388-95.

30. Noël G, Proudhom MA, Valery CA, et al. Radiosurgery for re-irradiation of brain metastasis: results in 54 patients. Radiother Oncol 2001;60:61-7.

31. Gaspar L, Scott C, Rotman M, et al. Recursive partitioning analysis (RPA) of prognostic factors in three Radiation Therapy Oncology Group (RTOG) brain metastases trials. Int J Radiat Oncol Biol Phys 1997;37:745-51.

32. Sperduto PW, Berkey B, Gaspar LE, et al. A new prognostic index and comparison to three other indices for patients with brain metastases: an analysis of 1,960 patients in the RTOG database. Int J Radiat Oncol Biol Phys 2008;70:510-4. 
33. Sperduto PW, Kased N, Roberge D, et al. Summary report on the graded prognostic assessment: an accurate and facile diagnosis-specific tool to estimate survival for patients with brain metastases. J Clin Oncol 2012;30:419-25.

34. Faithfull S, Brada M. Somnolence syndrome in adults following cranial irradiation for primary brain tumours. Clin Oncol (R Coll Radiol) 1998;10:250-4.

35. Soussain C, Ricard D, Fike JR, et al. CNS complications of radiotherapy and chemotherapy. Lancet 2009;374:1639-51.

36. Hirsch FR, Suda K, Wiens J, et al. New and emerging

Cite this article as: Keller A, Lefebvre F, Ricard D, Noël G, Antoni D. Outcomes of adjuvant whole-brain radiotherapy versus hypofractionated stereotactic radiotherapy after surgical resection of brain metastases: a propensity score-matched analysis. Chin Clin Oncol 2020;9(4):55. doi: 10.21037/cco-19-269 targeted treatments in advanced non-small-cell lung cancer. Lancet 2016;388:1012-24.

37. Chen RC, Gabriel PE, Kavanagh BD, et al. How Will Big Data Impact Clinical Decision Making and Precision Medicine in Radiation Therapy? Int J Radiat Oncol Biol Phys 2016;95:880-4.

38. McNutt TR, Moore KL, Quon H. Needs and Challenges for Big Data in Radiation Oncology. Int J Radiat Oncol Biol Phys 2016;95:909-15. 